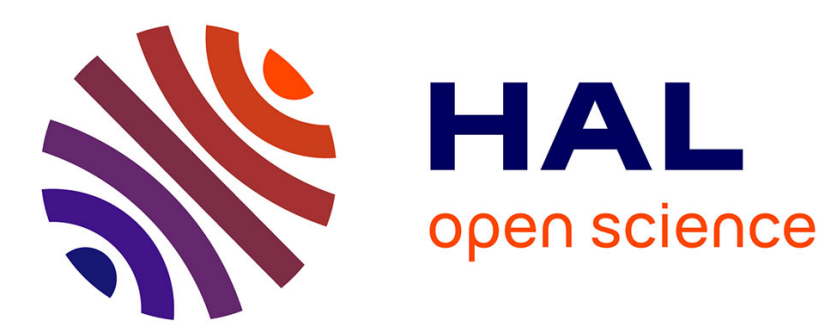

\title{
SUR LES VARIÉTÉS LORENTZIENNES DONT LE GROUPE CONFORME EST ESSENTIEL
}

\author{
Charles Frances
}

\section{To cite this version:}

Charles Frances. SUR LES VARIÉTÉS LORENTZIENNES DONT LE GROUPE CONFORME EST ESSENTIEL. Mathematische Annalen, 2002, 332 (1), pp.103-119. 10.1007/s00208-004-0619-x . hal03195438

\section{HAL Id: hal-03195438 \\ https://hal.science/hal-03195438}

Submitted on 11 Apr 2021

HAL is a multi-disciplinary open access archive for the deposit and dissemination of scientific research documents, whether they are published or not. The documents may come from teaching and research institutions in France or abroad, or from public or private research centers.
L'archive ouverte pluridisciplinaire HAL, est destinée au dépôt et à la diffusion de documents scientifiques de niveau recherche, publiés ou non, émanant des établissements d'enseignement et de recherche français ou étrangers, des laboratoires publics ou privés. 


\title{
SUR LES VARIÉTÉS LORENTZIENNES DONT LE GROUPE CONFORME EST ESSENTIEL
}

\author{
CHARLES FRANCES
}

\begin{abstract}
Nous proposons de nombreuses constructions de variétés compactes lorentziennes pour lesquelles le groupe conforme ne préserve aucune mesure lisse. Ceci montre que le théorème de Ferrand-Obata ne se généralise pas au cadre lorentzien.
\end{abstract}

\section{INTRODUCTION}

Un des premiers exemples d'espace-temps proposé comme solution de l'équation d'Einstein avec constante cosmologique positive, fut le produit $\mathbf{R} \times \mathbf{S}^{3}$ muni de la métrique $-d t^{2}+g_{\text {can }}$ (où $g_{\text {can }}$ désigne la métrique canonique de la sphère). On le baptisa univers statique d'Einstein. Son caractère statique, peu en accord avec les observations ultérieures, n'en font pas un modèle cosmologique réaliste, mais ceci n'affecte en rien son intérêt du point de vue géométrique. En effet, la structure conforme de cet espace, ou plus exactement du quotient de $\mathbf{S}^{1} \times \mathbf{S}^{n-1}$ (toujours muni de la métrique $\left.-d t^{2}+g_{\text {can }}\right)$ par le produit des antipodies, que l'on note $\mathbf{E i n}_{n}$, possède des propriétés remarquables, qui en font l'analogue conforme lorentzien de la sphère $\mathbf{S}^{n}$. C'est d'ailleurs ce quotient que nous nommerons univers d'Einstein. Parmi les analogies avec la sphère, on peut commencer par dire que $\mathbf{E i n}_{n}$ apparaît comme le bord conforme de $\mathbf{A d S _ { n + 1 }}$, l'espace antide Sitter de dimension $n+1$, qui est l'espace modèle lorentzien à courbure constante égale à -1. D'autre part, il existe sur $\operatorname{Ein}_{n}$ un analogue de la projection stéréographique qui identifie conformément l'espace de Minkowski $\mathbf{R}^{1, n-1}$ à un ouvert de $\mathbf{E i n}_{n}$ (plus généralement, tous les espaces modèles lorentziens à courbure constante sont conformément équivalents à un ouvert de $\left.\operatorname{Ein}_{n}\right)$. Ainsi, l'univers d'Einstein est conformément plat. On dispose également d'un analogue du théorème de Liouville pour les applications conformes de $\mathbf{E i n}_{n}$, qui affirme que toute transformation conforme locale est la restriction d'une unique transformation globale. Enfin, un dernier point commun entre la sphère conforme canonique et l'univers d'Einstein, est que ces deux espaces admettent beaucoup de symétries conformes : leurs groupes conformes sont respectivement $P O(1, n+1)$ pour $\mathbf{S}^{n}$, et $P O(2, n)$ pour $\mathbf{E i n}_{n}$. On peut voir $P O(2, n)$ comme le groupe de Möbius lorentzien.

Date: 29 Novembre 2002. 
Dans [D'AG], les auteurs font remarquer que génériquement, les structures géométriques rigides n'ont pas de symétries, et que par conséquent, celles qui en possèdent beaucoup (en un sens à préciser) doivent être suffisamment exceptionnelles pour que l'on puisse les "classifier". Ils proposent une belle illustration de ce principe pour les structures conformes riemanniennes via le :

[Ferrand-Obata [Ob] [Fe2]]

Si une variété compacte $M$ de dimension $n \geq 2$ est munie d'une structure conforme riemannienne $S$ pour laquelle le groupe conforme est non compact, alors $M$ est la sphère $\mathbf{S}^{n}$ et la structure $S$ est la structure conforme canonique.

On peut énoncer ce théorème en remplaçant "groupe conforme non compact" par "groupe conforme essentiel", essentiel signifiant que le groupe conforme ne préserve aucune métrique de la classe conforme considérée. Remarquons que cette définition d'essentialité a un sens pour n'importe quelle structure conforme pseudo-riemannienne. Il n'est pas difficile de vérifier que pour une variété riemannienne compacte, dire que le groupe conforme est essentiel équivaut à dire qu'il n'est pas compact.

Il est naturel de se demander si le théorème de Ferrand-Obata se généralise aux structures conformes pseudo-riemanniennes de signature supérieure. Dans le cas de la signature lorentzienne, par exemple, est-il vrai, comme cela fut brièvement conjecturé dans [D'AG], que les seules variétés compactes lorentziennes pour lesquelles le groupe conforme est essentiel sont, à quotients et revêtements finis près, conformément équivalentes à l'univers d'Einstein?

Notre but dans cet article est de répondre négativement à cette question, et de construire de nombreux exemples qui montrent que contrairement au cadre riemannien, il existe une relative abondance de variétés compactes lorentziennes pour lesquelles le groupe conforme est essentiel. On peut résumer cela par le

Pour tout entier $n \geq 3$ et tout entier $g \geq 1$, la variété produit du cercle $\mathbf{S}^{1}$ par la somme connexe de $g$ copies de $\mathbf{S}^{1} \times \mathbf{S}^{n-2}$ peut être munie d'une infinité de structures conformes lorentziennes essentielles non équivalentes. Deux structures sont dites non-équivalentes s'il n'existe aucun difféomorphisme conforme entre elles.

Les premiers exemples illustrant le théorème précédent vont être obtenus comme des variétés kleiniennes, c'est-à-dire des quotients $\Omega / \Gamma$ où $\Omega$ est un ouvert de $\widetilde{\operatorname{Ein}}_{n}$ (le produit $\mathbf{R} \times \mathbf{S}^{n-1}$ muni de la structure conforme associée à $-d t^{2}+g_{\text {can }}$ ) et $\Gamma$ un groupe discret de transformations conformes de $\widetilde{\operatorname{Ein}}_{n}$. Dans le cas de la dimension 3, nous montrons qu'il est possible de déformer des structures kleiniennes essentielles, pour obtenir des structures conformes lorentziennes qui ne sont plus kleiniennes mais restent essentielles. Nous obtenons le : 
Soit $\Sigma_{g}$ une surface orientable de genre $g(g \geq 2)$.

Il existe sur la variété $\mathbf{S}^{1} \times \Sigma_{g}$ une infinité de structures lorentziennes conformément plates non kleiniennes qui sont essentielles. Ces procédés rendent très hypothétique une classification globale des structures lorentziennes sur les variétés compactes pour lesquelles le groupe conforme est essentiel. Néanmoins, tous les exemples de structures essentielles que nous construisons dans cet articles ont la particularité d'être conformément plats, et l'on peut conjecturer, à l'instar de D'Ambra et Gromov dans [D'AG], que ce fait est général:

Conjecture. (Conjecture de Lichnerowicz généralisée)

Une variété lorentzienne compacte dont le groupe conforme est essentiel est conformément plate.

Contrairement au cadre riemannien, cette conjecture est fausse sans l'hypothèse de compacité : il existe des exemples de variétés lorentziennes non compactes, homogènes, non conformément plates, pour lesquelles le groupe conforme est essentiel (voir par exemple $[\mathrm{A}][\mathrm{KR}]$ ).

\section{GÉométrie De L'Univers D’Einstein}

Nous commençons par une brève description des principales propriétés géométriques de l'univers d'Einstein. Le lecteur intéressé par une étude plus détaillée pourra consulter $[\mathrm{P}],[\mathrm{HE}]$ ou $[\mathrm{Fr} 1]$.

2.1. La structure conforme canonique de l'espace $\operatorname{Ein}_{n}$. On désigne par $\mathbf{R}^{2, n}$ l'espace $\mathbf{R}^{n+2}$ muni de la forme quadratique

$$
q^{2, n}=-2 x_{1} x_{n+2}-2 x_{2} x_{n+1}+x_{3}^{2}+\ldots+x_{n}^{2}
$$

et par $\pi$ la projection de $\mathbf{R}^{n+2}$ sur l'espace projectif $\mathbf{R P}^{n+1}$.

Le cône isotrope de la forme $q^{2, n}$, que l'on note $\mathbf{C}^{2, n}$, se projette par $\pi$ sur une hypersurface $\Sigma$ de $\mathbf{R P}^{n+1}$. Celle-ci est munie d'une structure conforme naturelle. Pour le voir, on commence par remarquer que pour tout $x \in$ $\mathbf{C}^{2, n}$, la restriction de $q^{2, n}$ à l'espace tangent en $x$ au cône est une forme quadratique dégénérée $\hat{q}_{x}^{2, n}$, de signature $(0,-,+\ldots+)$. Le noyau de cette forme quadratique est précisément la droite de $\mathbf{C}^{2, n}$ passant par 0 et par $x$ et il coïncide avec le noyau de l'application $d_{x} \pi$. Si $\hat{C}_{x}$ désigne le cône isotrope de $\hat{q}_{x}^{2, n}$, alors $d_{x} \pi\left(\hat{C}_{x}\right)$ est un cône sur l'espace tangent à $\Sigma$ en $\pi(x)$ qui est défini par une forme quadratique lorentzienne. De plus, si $x$ et $y$ sont dans la même fibre de $\pi$, on a $d_{x} \pi\left(\hat{C}_{x}\right)=d_{y} \pi\left(\hat{C}_{y}\right)$. Ainsi, on vient de définir sur $\Sigma$ une distribution lisse de cônes tangents lorentziens, ou de manière équivalente, une classe conforme de métriques lorentziennes. On appelle univers d'Einstein, noté $\mathbf{E i n}_{n}$, l'hypersurface $\Sigma$ munie de cette classe conforme canonique. Si l'on se place dans une base $\left(e_{1}^{\prime}, \ldots, e_{n+2}^{\prime}\right)$ où $q^{2, n}$ s'exprime par $-y_{1}^{2}-y_{2}^{2}+y_{3}^{2}+\ldots+y_{n+2}^{2}$. Alors l'intersection de $\mathbf{C}^{2, n}$ avec la sphère euclidienne de rayon 2 (dans la nouvelle base), que l'on note 
$\widehat{\operatorname{Ein}}_{n}$, est topologiquement $\mathbf{S}^{1} \times \mathbf{S}^{n-1}$. La métrique que $q^{2, n}$ induit sur $\widehat{\operatorname{Ein}}_{n}$ est $-d t^{2}+g_{c a n}\left(g_{c a n}\right.$ désigne la métrique canonique sur $\left.\mathbf{S}^{n-1}\right)$. L'univers d'Einstein étant le quotient de $\widehat{\mathbf{E i n}}_{n}$ par le produit des antipodies, c'est un fibré en $\mathbf{S}^{n-1}$ sur $\mathbf{R} \mathbf{P}^{1}$.

Comme la structure conforme sur $\operatorname{Ein}_{n}$ est construite naturellement à partir de la métrique $q^{2, n}$, il est clair que toute transformation de $P O(2, n)$ agit conformément sur $\operatorname{Ein}_{n}$. Réciproquement, on a ([CK] [Fr1]) :

Le groupe conforme de l'univers d'Einstein coïncide avec $P O(2, n)$.

Dans les deux références sus-citées, on trouve également une version du théorème de Liouville pour l'univers d'Einstein :

[Liouville] Tout difféomorphisme conforme entre ouverts de $\mathbf{E i n}_{n}$ est la restriction d'un unique élément de $P O(2, n)$.

2.2. Géodésiques et cônes de lumière. On appelle géodésiques de lumière de $\mathbf{E i n}_{n}$ les projetés sur $\mathbf{E i n}_{n}$ des plans isotropes de $\mathbf{R}^{2, n}$ (c'està-dire les plans où la forme quadratique $q^{2, n}$ est nulle). Les géodésiques de lumière sont donc des cercles et toute transformation conforme de $\mathbf{E i n}_{n}$ envoie géodésique de lumière sur géodésique de lumière. Notons que contrairement aux géodésiques riemanniennes ou lorentziennes, il n'y a pas de paramétrage privilégié pour ces courbes.

Soit maintenant $p$ un point de $\operatorname{Ein}_{n}$. On appelle cône de lumière de sommet $\mathbf{p}$ l'ensemble des géodésiques de lumière qui passent par $p$, et on le note $C(p)$. Le cône $C(p)$ n'est pas lisse en $p$. L'hypersurface $C(p) \backslash\{p\}$ est conformément équivalente au cylindre $\mathbf{R} \times \mathbf{S}^{n-2}$ muni de la métrique singulière $0+g_{\text {can }}$.

2.3. Composantes de Minkowski, composantes de Sitter et anti-de Sitter. L'univers d'Einstein peut se voir comme la compactification conforme de l'espace de Minkowski par un cône de lumière. En effet: Pour tout point $p$ de $\operatorname{Ein}_{n}$, le complémentaire de $C(p)$ dans $\operatorname{Ein}_{n}$ est un ouvert conformément équivalent à l'espace de Minkowski $\mathbf{R}^{1, n-1}$. On peut trouver une preuve de ce fait dans [P] ou [Fr1], ainsi qu'une description du comportement "à l'infini" des droites de l'espace de Minkowski. Par la suite, nous appellerons projection stéréographique de pôle $p$ tout difféomorphisme conforme entre le complémentaire d'un cône de lumière et $\mathbf{R}^{1, n-1}$.

Des procédés de compactification similaires existent pour les espaces de Sitter et anti-de Sitter. Par exemple, l'espace anti-de Sitter, défini par $-2 x_{1} x_{n+2}-2 x_{2} x_{n+1}+x_{3}^{2}+\ldots+x_{n}^{2}=-1$, peut se voir comme le projeté des points de $C^{2, n}$ pour lesquels $x_{n} \neq 0$. Son complémentaire dans $\operatorname{Ein}_{n}$ est juste le projeté de l'intersection $e_{n}^{\perp} \cap \mathbf{C}^{2, n}$ ( $e_{n}^{\perp}$ est l'orthogonal de $e_{n}$ pour la forme $\left.q^{2, n}\right)$, c'est à dire un univers d'Einstein de codimension 1. Par homogénéité de l'univers d'Einstein, on obtient donc que le complémentaire dans $\mathbf{E i n}_{n}$ de tout univers d'Einstein de codimension 1 est conformément équivalent à 
l'espace anti-de Sitter. On montre de manière analogue que lorsque $v$ est un vecteur de type temps dans $\mathbf{R}^{2, n}$, le projeté de $v^{\perp} \cap \mathbf{C}^{2, n} \operatorname{sur}_{\mathbf{E i n}_{n}}$ est une sphère riemannienne de codimension 1 , dont le complémentaire est un ouvert conformément équivalent à l'espace de Sitter de dimension $n$.

2.4. Complémentaire d'une géodésique de lumière. Étant donnée une géodésique de lumière $\Delta_{0}$ de l'univers d'Einstein, nous appelons $\Omega_{\Delta_{0}}$ l'ouvert $\operatorname{Ein}_{n} \backslash \Delta_{0}$. Nous disons ici quelques mots de la géométrie de ces ouverts, géométrie qui nous sera utile en section 4.3.

On commence par remarquer que pour tout $x$ sur $\Delta_{0}$, l'intersection du cône $C(x)$ avec $\Omega_{\Delta_{0}}$ est $C(x) \backslash \Delta_{0}$, c'est à dire une hypersurface dégénérée de $\Omega_{\Delta_{0}}$ difféomorphe à $\mathbf{R}^{n-1}$. On note cette hypersurface $H_{\Delta_{0}}(x)$. Remarquons que si $x \neq x^{\prime}$, alors $H_{\Delta_{0}}(x)$ et $H_{\Delta_{0}}\left(x^{\prime}\right)$. En effet, il n'existe pas dans $\operatorname{Ein}_{n}$, de triangle non trivial dont les côtés soient des segments géodésiques de lumière (si c'était le cas, $q^{2, n}$ serait nulle sur des sous-espace de dimension 3 dans $\left.\mathbf{R}^{2, n}\right)$. Les hypersurfaces $H_{\Delta_{0}}(x)$ constituent donc un feuilletage de $\Omega_{\Delta_{0}}$ que l'on appelle $H_{\Delta_{0}}$. On a de plus:

Il existe un difféomorphisme entre $\Omega_{\Delta_{0}}$ et $\mathbf{S}^{1} \times \mathbf{R}^{n-1}$ qui envoie les feuilles de $H_{\Delta_{0}}$ sur les hypersurfaces $\{\theta\} \times \mathbf{R}^{n-1}$.

Proof. On travaille dans la base $\left(e_{1}, \ldots, e_{n+2}\right)$ et on prend pour $\Delta_{0}$ la projection $\operatorname{sur} \operatorname{Ein}_{n}$ du plan engendré par $e_{1}$ et $e_{2}$. Soit $x_{0}$ un point sur $\Delta_{0}$. On note $H_{0}$ la feuille de $H_{\Delta_{0}}$ issue de $x_{0}$. On choisit un difféomorphisme $\phi$ de $\mathbf{R}^{n-1}$ sur $H_{0}$. Si $A$ est une matrice de $S L_{2}(\mathbf{R})$, la matrice

$$
R_{A}=\left(\begin{array}{ccc}
A & & \\
& I d_{n-2} & \\
& & A
\end{array}\right)
$$

de $O(2, n)$ correspond à une transformation conforme de $\mathbf{E i n}_{n}$ préservant $\Omega_{\Delta_{0}}$. Considérons maintenant $A(\theta)$ un groupe à un paramètre de rotations dans $S L_{2}(\mathbf{R})$, et $R_{A(\theta)}$ le groupe à un paramètre d'applications conformes de $\Omega_{\Delta_{0}}$ associé. Il suffit alors de considérer le difféomorphisme suivant pour prouver le lemme :

$$
\begin{array}{rlc}
\psi: \mathbf{S}^{1} \times \mathbf{R}^{n-1} & \rightarrow & \Omega_{\Delta_{0}} \\
(\theta, x) & \mapsto & R_{A(\theta)}(\phi(x))
\end{array}
$$

\section{Dynamique COnforme Sur L'Univers D'Einstein}

Le groupe de Möbius lorentzien possède une dynamique bien plus riche que son analogue riemannien. Nous avons mené l'étude détaillée de cette dynamique dans [Fr2] mais ici, nous nous limitons aux quelques propriétés dynamiques utiles à la preuve du théorème 1 . 
3.1. Décomposition de Cartan du groupe $O(2, n)$. Nous rappelons que $O(2, n)$ désigne le sous-groupe de $G L_{n+2}(\mathbf{R})$ qui préserve la forme quadratique $q^{2, n}(x)=-2 x_{1} x_{n+2}+2 x_{2} x_{n+1}+x_{3}^{2}+\ldots+x_{n}^{2}$.

On désigne par $A^{+}$le groupe des matrices diagonales de $S O(2, n)$ qui sont de la forme

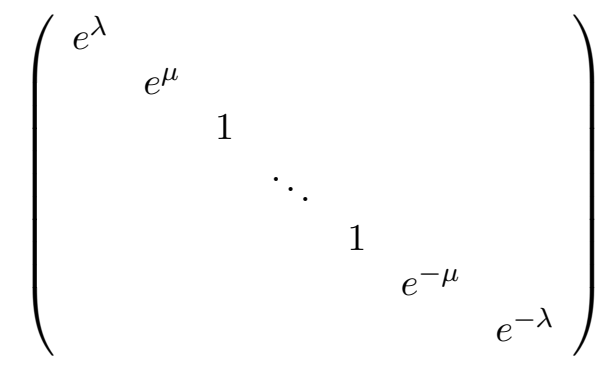

avec $\lambda \geq \mu \geq 0$.

Il existe alors un sous-groupe compact maximal $K$ tel que $S O(2, n)$ s'écrive sous la forme $K A^{+} K$ ([B], [IW]). Si l'on choisit un élément $g_{0}$ dans $O(2, n)$ qui ne soit pas dans $S O(2, n)$, alors quitte à remplacer $K$ par $K \cup g_{0} K$, on obtient que $O(2, n)$ s'écrit également sous la forme $K A^{+} K$ (attention, $K$ est toujours compact mais ce n'est plus un groupe). Cette décomposition s'appelle décomposition de Cartan du groupe $O(2, n)$.

Pour tout $g \in O(2, n)$, il existe un unique $a(g) \in A^{+}$tel que $g \in K a(g) K$. L'élément $a(g)$ s'appelle la projection de Cartan de $g$. Matriciellement, il s'écrit sous la forme :

$$
a(g)=\left(\begin{array}{ccccccc}
e^{\lambda(g)} & & & & & & \\
& e^{\mu(g)} & & & & & \\
& & 1 & & & & \\
& & & \ddots & & & \\
& & & 1 & & \\
& & & & & e^{-\mu(g)} & \\
& & & & & & e^{-\lambda(g)}
\end{array}\right)
$$

Les réels $\lambda(g)$ et $\mu(g)$ s'appellent les distorsions de l'élément $g$ (associées à la décomposition de Cartan considérée).

Si $\left(g_{k}\right)$ est une suite d'éléments de $O(2, n)$ qui tend vers l'infini, on note $\lambda_{k}=\lambda\left(g_{k}\right), \mu_{k}=\mu\left(g_{k}\right)$ et $\delta_{k}=\lambda_{k}-\mu_{k}$.

On dit que la suite $\left(g_{k}\right)$ tend simplement vers l'infini lorsque les suites $\left(\lambda_{k}\right),\left(\mu_{k}\right)$ et $\left(\delta_{k}\right)$ admettent toutes trois des limites dans $\overline{\mathbf{R}}$ que l'on note $\lambda_{\infty}, \mu_{\infty}$ et $\delta_{\infty}$ et que de plus, les facteurs compacts de la décomposition de Cartan de $\left(g_{k}\right)$ admettent tous les deux une limite dans $K$. On remarque que toute suite qui tend vers l'infini admet une suite extraite tendant simplement vers l'infini.

3.2. Dynamique à distorsions équilibrées. On dit qu'une suite $\left(g_{k}\right)$ de $O(2, n)$ qui tend simplement vers l'infini est à distorsions équilibrées 
lorsque $\lambda_{\infty}=\mu_{\infty}=+\infty$ et $\delta_{\infty}$ est fini. Les propriétés dynamiques d'une telle suite sont résumées par la :

Soit $\left(g_{k}\right)$ une suite de $O(2, n)$ à distorsions équilibrées. On peut lui associer deux géodésiques de lumière $\Delta^{+}$et $\Delta^{-}$appelés cercles attracteurs et répulseurs de $\left(g_{k}\right)$, ainsi que deux submersions $\pi_{+}: \Omega_{\Delta^{-}} \rightarrow \Delta^{+}$(resp. $\pi_{-}: \Omega_{\Delta^{+}} \rightarrow \Delta^{-}$) dont les fibres sont les feuilles de $H_{\Delta^{-}}\left(\right.$resp. $\left.H_{\Delta^{+}}\right)$de sorte que:

(i) Pour tout $x_{\infty} \in \Omega_{\Delta^{-}}$, et toute suite de points $\left(x_{k}\right)$ qui converge vers $x_{\infty}$, la suite $g_{k}\left(x_{k}\right)$ converge vers $\pi_{+}\left(x_{\infty}\right)$.

(ii) Soit $U$ un petit voisinage tubulaire (ouvert) de $\Delta^{-}$et $U^{c}$ son complémentaire dans $\operatorname{Ein}_{n}$. Alors $\lim _{k \rightarrow \infty} g_{k}\left(U^{c}\right)=\Delta^{+}$.

Proof. Il suffit de prouver la propriété pour une suite $\left(g_{k}\right)$ de $A^{+}$. Pour tout $x=\left(x_{1}, x_{2}, \ldots, x_{n+2}\right)$ dans $\mathbf{R}^{2, n}$, on pose $B_{\epsilon}(x)=\left[x_{1}-\epsilon, x_{1}+\epsilon\right] \times\left[x_{2}-\right.$ $\left.\epsilon, x_{2}+\epsilon\right] \times \ldots \times\left[x_{n+2}-\epsilon, x_{n+2}+\epsilon\right]$. On note $\Delta^{+}$la projection sur $\operatorname{Ein}_{n} \mathrm{du}$ plan $P^{+}$engendré par $e_{1}$ et $e_{n+2}$ et $\Delta^{-}$celle du plan $P^{-}$engendré par $e_{2}$ et $e_{n+1}$.

Si l'on choisit $x$ tel que $\pi(x) \notin \Delta^{-}$alors

$\lim _{k \rightarrow \infty} g_{k} \circ \pi\left(B_{\epsilon}(x)\right)=B_{\epsilon}^{\infty}(x)=\pi\left(\left[x_{1}-\epsilon, x_{1}+\epsilon\right] \times\left[x_{2}-\epsilon, x_{2}+\epsilon\right] \times\{0\} \times \ldots . . \times\{0\}\right)$

Ainsi, $B_{\epsilon}^{\infty}(x)$ est un petit morceau du cercle $\Delta^{+}$. En faisant tendre $\epsilon$ vers 0 , on voit que toute suite $\pi\left(x_{k}\right)$ qui tend vers $\pi(x)$ vérifie $\lim _{k \rightarrow \infty} g_{k} \circ \pi\left(x_{k}\right)=$ $\lim _{k \rightarrow \infty} g_{k} \circ \pi(x)=\pi\left(x_{1}, x_{2}, 0, \ldots, 0\right)$. On appelle $\tilde{\pi}^{+}$la projection de $\mathbf{R}^{2, n}$ sur le plan $P^{+}$. Elle induit une projection $\pi^{+}$de $\operatorname{Ein}_{n} \backslash \Delta^{-}=\Omega_{\Delta^{-}}$sur $\Delta^{+}$ dont les fibres sont les projetés sur $\operatorname{Ein}_{n}$ des fibres de $\tilde{\pi}^{+}$. Ces dernières sont des hyperplans dégénérés de $\mathbf{R}^{2, n}$, obtenus comme les orthogonaux des vecteurs de $P^{-}$pour la forme $q^{2, n}$. Les fibres de $\pi^{+}$sont donc les cônes de lumière issus des points de $\Delta^{-}$, autrement dit, ce sont les feuilles du feuilletage $H_{\Delta^{-}}$. Ceci prouve le point $(i)$.

Pour montrer le point (ii), on recouvre $U^{c}$ par un nombre fini de $\pi\left(B_{\epsilon}\right)$, et on voit que $\lim _{k \rightarrow+\infty} g_{k}\left(U^{c}\right)$ est un morceau de $\Delta^{+}$. Mais comme $U^{c}$ rencontre toutes les fibres de $\pi^{+}$, on obtient qu'en fait cette limite est l'intégralité de $\Delta^{+}$.

Soit $\left(g_{k}\right)$ une suite de $P O(2, n)$ qui tend simplement vers l'infini. On suppose que cette suite laisse invariant un ouvert $\Omega \subset \operatorname{Ein}_{n}$. On dit que $\left(g_{k}\right)$ agit proprement sur $\Omega$ si pour tout compact $K \subset \Omega$, on a $g_{k}(K) \cap K=$ $\emptyset$, sauf éventuellement pour une nombre fini d'indices $k$. De la proposition précédente, on déduit facilement le : Si une suite $\left(g_{k}\right)$ à distorsions équilibrées, de cercles attracteurs et répulseurs $\Delta^{+}$et $\Delta^{-}$, agit sur un ouvert $\Omega \subset \operatorname{Ein}_{n}$, et que $\Omega$ ne rencontre ni $\Delta^{+}$, ni $\Delta^{-}$, alors $\left(g_{k}\right)$ agit proprement $\operatorname{sur} \Omega$.

\section{Construction de variétés COMPaCtes essentielles}

4.1. Structures lorentziennes conformément plates. On dit qu'une structure lorentzienne sur une variété $M$ est conformément plate si elle est 
localement conformément équivalente à la structure conforme de l'espace de Minkowski $\mathbf{R}^{1, n-1}$. On note $\tilde{M}$ le revêtement universel de $M$, que l'on munit de la structure conforme obtenue en remontant celle de $M$. L'existence d'un théorème de Liouville lorentzien et de projections stéréographiques assurent qu'en dimension $n \geq 3$, une structure conformément plate équivaut à la donnée d'une $\left(P O(2, n), \mathbf{E i n}_{n}\right)$-structure sur la variété $M$. Rappelons qu'à une telle structure sont associés une application développante $\delta$, qui est une immersion conforme de $\tilde{M}$ dans $\mathbf{E i n}_{n}$, et un morphisme d'holonomie $\rho$ : $\pi_{1}(M) \rightarrow P O(2, n)$ vérifiant, pour tout $\gamma \in \pi_{1}(M)$, la relation d'équivariance $\delta \circ \gamma=\rho(\gamma) \circ \delta$. On appelle $H=\rho\left(\pi_{1}(M)\right)$ le groupe d'holonomie de la structure conformément plate considérée.

On désigne par $\widetilde{\operatorname{Ein}}_{n}$ le produit $\mathbf{R} \times \mathbf{S}^{n-1}$ muni de la classe conforme associée à la métrique $-d t^{2}+g_{c a n}\left(g_{c a n}\right.$ est la métrique canonique de $\left.\mathbf{S}^{n-1}\right)$.

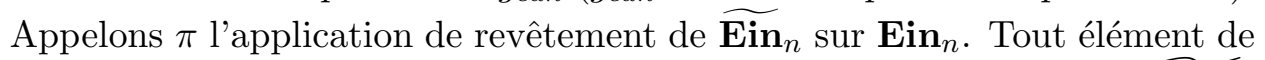
$P O(2, n)$ se relève en une application conforme de $\widetilde{\operatorname{Ein}}_{n}$, et on note $\widetilde{O(2, n)}$ le remonté de tout le groupe $P O(2, n)$. On obtient un morphisme naturel $\bar{\pi}$ de $\widetilde{O(2, n)}$ sur $P O(2, n)$. Toute structure conformément plate sur $M$ peut également s'interpréter comme une $\left(\widetilde{O(2, n)}, \widetilde{\operatorname{Ein}}_{n}\right)$-structure, d'application développante $\tilde{\delta}$ et de morphisme d'holonomie $\tilde{\rho}$, tels que $\pi \circ \tilde{\delta}=\delta$ et $\bar{\pi} \circ \tilde{\rho}=\rho$.

Des exemples particuliers de structures conformément plates sont les structures kleiniennes obtenues comme des quotient $\tilde{\Omega} / \tilde{\Gamma}$, où $\tilde{\Omega}$ est un ouvert de $\widetilde{\operatorname{Ein}}_{n}$ et $\tilde{\Gamma}$ un groupe discret de $\widetilde{O(2, n)}$ agissant proprement discontinûment sur $\tilde{\Omega}$. Pour de telles structures, l'application $\tilde{\delta}$ est un revêtement sur son image, et le groupe d'holonomie $\tilde{\rho}\left(\pi_{1}(M)\right)$ coïncide avec $\tilde{\Gamma}$; en particulier il est discret. Bien sûr, toute structure $\Omega / \Gamma$ avec $\Omega$ ouvert de $\operatorname{Ein}_{n}$ et $\Gamma$ groupe discret de $P O(2, n)$ agissant proprement discontinûment sur $\Omega$ est un cas particulier de structure kleinienne. Nous allons tout de suite en construire des exemples.

4.2. Des groupes de Schottky lorentziens. [Groupes de Schottky de $\left.S L_{2}(\mathbf{R})\right]$ On dit qu'un groupe $\hat{\Gamma} \subset S L_{2}(\mathbf{R})$ engendré par $g$ éléments $\hat{\gamma}_{1}, \ldots, \hat{\gamma}_{g}$ est un groupe de Schottky lorsqu'il existe $2 g$ demi-disques fermés de $\mathbf{H}^{2}$, notés $D_{1}^{+}, \ldots, D_{g}^{+}, D_{1}^{-}, \ldots, D_{g}^{-}$, qui sont deux à deux disjoints, et tels que pour tout $i \in\{1, \ldots, g\}: \gamma_{i}\left(\mathbf{H}^{2} \backslash D_{i}^{-}\right)=\overline{D_{i}^{+}}$. Rappelons (voir par exemple [Ma]) qu'un groupe de Schottky de $S L_{2}(\mathbf{R})$ est toujours constitué d'éléments hyperboliques.

On travaille encore avec la base $\left(e_{1}, \ldots, e_{n+2}\right)$ dans laquelle $q^{2, n}$ s'exprime par $q^{2, n}(x)=-2 x_{1} x_{n+2}+2 x_{2} x_{n+1}+x_{3}^{2}+\ldots+x_{n}^{2}$ et on appelle $\mathbf{T}_{0}$ le tore d'Einstein obtenu comme projection sur $\mathbf{E i n}_{n}$ du sous-espace vect $\left(e_{1}, e_{2}, e_{n+1}, e_{n+2}\right)$. Ce tore est conformément le produit $\mathbf{S}^{1} \times \mathbf{S}^{1}$ muni de la métrique $d x d y$. Il existe donc sur $\mathbf{T}_{0}$ deux feuilletages par géodésiques de lumière. Le premier, noté $F_{1}$ a pour feuilles les $\{x\} \times \mathbf{S}^{1}$ et le second $F_{2}$ est constitué des 
$\mathbf{S}^{1} \times\{y\}$

On considère les deux représentations $\rho_{D}$ et $\rho_{G}$ de $S L_{2}(\mathbf{R})$ dans $O(2, n)$ définies, pour tout $A=\left(\begin{array}{ll}a & b \\ c & d\end{array}\right)$ dans $S L_{2}(\mathbf{R})$, de la manière suivante :

$$
\rho_{G}(A)=\left(\begin{array}{ccc}
A & & \\
& I_{n-2} & \\
& & A
\end{array}\right)
$$

et

$$
\rho_{D}(A)=\left(\begin{array}{ccc}
a I_{2} & & b I_{2} \\
& I_{n-2} & \\
c I_{2} & & d I_{2}
\end{array}\right)
$$

Notons que $\rho_{G}(A)$ (resp. $\rho_{D}(A)$ ) préserve $\mathbf{T}_{0}=\mathbf{S}^{1} \times \mathbf{S}^{1}$ en agissant projectivement par $A$ sur le facteur de gauche (resp. le facteur de droite) et trivialement sur l'autre.

Considérons à présent un groupe de Schottky $\hat{\Gamma}$ dans $S L_{2}(\mathbf{R})$ engendré par $g$ générateurs $\hat{s}_{1}, \ldots, \hat{s}_{g}$. On appelle $\Gamma$ le groupe $\rho_{G}(\hat{\Gamma})$, et on pose $s_{i}=\rho_{G}\left(\hat{s}_{i}\right)$.

Si $\hat{\gamma}$ est un élément hyperbolique de $S L_{2}(\mathbf{R})$, alors l'élément $\gamma=\rho_{G}(\hat{\gamma})$ s'écrit dans une certaine base sous la forme

$$
\left(\begin{array}{lllll}
\lambda & & & & \\
& \lambda^{-1} & & & \\
& & I_{n-2} & & \\
& & & \lambda & \\
& & & & \lambda^{-1}
\end{array}\right)
$$

et par conséquent $\left(\gamma^{k}\right)$ est à distorsions équilibrées. Ses cercles attracteurs et répulseurs sont disjoints et appartiennent au feuilletage $F_{1}$ de $\mathbf{T}_{0}$. Ils correspondent aux points fixes de $\hat{\gamma}$ sur $\mathbf{S}^{1}$. Ainsi, pour tout $i \in\{1, \ldots, g\}$, on note $\Delta_{i}^{+}$et $\Delta_{i}^{-}$les cercles attracteurs et répulseurs de la suite $\left(s_{i}^{k}\right)$. Ils sont tous disjoints deux à deux puisque les pôles des $\hat{s}_{i}$ sont eux-même disjoints deux à deux. Maintenant, d'après la proposition 3.2 (et quitte à remplacer $s_{i}$ par une certaine puissance $s_{i}^{m_{i}}$ ), on peut choisir pour chaque $i$ de $\{1, \ldots, g\}$ un voisinage précompact ouvert $U_{i}^{+}$(resp. $U_{i}^{-}$) de $\Delta_{i}^{+}$(resp. $\left.\Delta_{i}^{-}\right)$de sorte que :

(i) Les $U_{i}^{ \pm}$sont d'adhérences disjointes deux à deux.

(ii) Pour tout $i$ de $\{1, \ldots, g\}, s_{i}\left(\operatorname{Ein}_{n} \backslash \overline{U_{i}^{-}}\right)=U_{i}^{+}$et $s_{i}^{-1}\left(\operatorname{Ein}_{n} \backslash \overline{U_{i}^{+}}\right)=U_{i}^{-}$.

Autrement dit, le groupe $\Gamma$ admet une dynamique de type ping-pong sur $\operatorname{Ein}_{n}$ (voir par exemple [dlH], [Ma] pour ce type de dynamique). On dit que c'est un groupe de Schottky de $P O(2, n)$. Nous définissons l'ensemble limite $\Lambda_{\Gamma}$ de $\Gamma$ comme le fermé de $\mathbf{T}_{0}$ donné par $\Lambda_{\hat{\Gamma}} \times \mathbf{S}^{1}$, où $\Lambda_{\hat{\Gamma}}$ est l'ensemble limite de $\hat{\Gamma}$ sur le cercle. Nous montrons alors :

Soit $\Omega_{\Gamma}=\operatorname{Ein}_{n} \backslash \Lambda_{\Gamma}$. Le groupe $\Gamma$ agit proprement discontinûment sur $\Omega_{\Gamma}$, et son action est cocompacte. 
Proof. On appelle $D$ le complémentaire dans $\operatorname{Ein}_{n}$ de la réunion des $U_{i}^{ \pm}$. Il n'est pas difficile de vérifier que $\bigcup_{\gamma \in \Gamma} \gamma(D)$ est un ouvert $\Omega$, stable par $\Gamma$. D'autre part, en regardant l'intersection de $D$ avec $\mathbf{T}_{0}$, et sa projection sur le facteur gauche de $\mathbf{S}^{1} \times \mathbf{S}^{1}$, on obtient un domaine fondamental $\hat{D}$ pour l'action de $\hat{\Gamma}$ sur le cercle. Ainsi $\hat{D}$ ne rencontre pas $\Lambda_{\hat{\Gamma}}$ et donc $D$ ne rencontre pas $\Lambda_{\Gamma}$. On en déduit que $\Omega$ est inclus dans $\Omega_{\Gamma}$. Maintenant, considérons $\left(\gamma_{k}\right)$, une suite de $\Gamma$ qui tend simplement vers l'infini. Alors $\gamma_{k}=$ $\rho_{G}\left(\hat{\gamma}_{k}\right)$ pour une suite $\left(\hat{\gamma}_{k}\right)$ tendant simplement vers l'infini dans $S L_{2}(\mathbf{R})$. Étant donnée l'expression matricielle de $\left(\gamma_{k}\right)$, cette suite est à distorsion équilibrée, et ses cercles attracteurs et répulseurs sont les cercles $\Delta^{+}=$ $\left\{p^{+}\right\} \times \mathbf{S}^{1}$ et $\Delta^{-}=\left\{p^{-}\right\} \times \mathbf{S}^{1}$, où $p^{+}$et $p^{-}$sont les pôles attracteurs et répulseurs (sur le cercle) de la suite $\left(\hat{\gamma}_{k}\right)$. Ainsi $\Delta^{+}$et $\Delta^{-}$sont dans $\Lambda_{\Gamma}$ et en appliquant le corollaire 3.2 , on obtient que $\Gamma$ agit proprement sur $\Omega_{\Gamma}$. Comme il agit cocompactement sur $\Omega$ ( $D$ est un domaine fondamental), on conclut $\Omega=\Omega_{\Gamma}$, ce qui achève la preuve.

4.3. Topologie des variétés quotients. Nous désignons par $\Gamma=\left\{s_{1}, \ldots, s_{g}\right\}$ un groupe de Schottky lorentzien comme dans la section précédente, et nous faisons l'hypothèse supplémentaire qu'il est contenu dans la composante connexe de l'identité. On note $M$ la variété compacte $\Omega_{\Gamma} / \Gamma$. En nous inspirant de l'étude des groupes de Schottky complexes faite par Seade et Verjovsky dans $[\mathrm{SV}]$, nous allons prouver le

La variété $M$ est difféomorphe au produit $\mathbf{S}^{1} \times\left(\mathbf{S}^{1} \times \mathbf{S}^{n-2}\right)^{(g-1) \sharp}$ où $\left(\mathbf{S}^{1} \times \mathbf{S}^{n-2}\right)^{(g-1) \sharp}$ désigne la somme connexe de $g-1$ copies de $\mathbf{S}^{1} \times \mathbf{S}^{n-2}$.

Proof. On désigne toujours par $\Delta_{i}^{+}$et $\Delta_{i}^{-}$les cercles attracteurs et répulseurs de $s_{i}$. Les voisinages tubulaires $U_{i}^{ \pm}$sont les mêmes que dans la section précédente et on appelle $\Sigma_{i}^{ \pm}$leurs frontières. Nous allons tout d'abord montrer

Toute géodésique de lumière de $\mathbf{E i n}_{n}$ admet une base de voisinages tubulaires dont la frontière est une hypersurface lisse de $\mathbf{E i n}_{n}$ de signature lorentzienne.

Proof. Dans le modèle $\widehat{\mathbf{E i n}}_{n}$ (voir section 2.1), il est clair que toute courbe de type $\theta \mapsto(\theta, x)$ admet une base de voisinages dont les frontières sont des hypersurfaces de signature lorentzienne. Il s'agit des voisinages $U_{\epsilon}=$ $\bigcup_{\theta \in \mathbf{S}^{1}}\left(\theta, D_{\epsilon}\right)$ avec $D_{\epsilon}$ la boule de centre $x$ et de rayon $\epsilon$ dans $\mathbf{S}^{n-1}$. Bien sûr, les projections de ces courbes sur $\operatorname{Ein}_{n}$, ainsi que leurs images par une transformation conforme ont la même propriété. On vient donc de montrer que les courbes, obtenues comme projection sur $\operatorname{Ein}_{n}$ des sous-espaces de $\mathbf{R}^{2, n}$ de dimension 3 et de signature $(--+)$, possèdent une base de voisinages avec la propriété requise. Le lemme découle maintenant du fait que toute géodésique de lumière peut être approximée par une telle courbe.

On peut donc supposer par la suite que les $\Sigma_{i}^{ \pm}$sont des hypersurfaces lisses de $\mathbf{E i n}_{n}$ dont la signature est lorentzienne. 
On considère maintenant l'ouvert $\Omega_{\Delta_{1}^{-}}=\operatorname{Ein}_{n} \backslash \Delta_{1}^{-}$. Rappelons que le feuilletage $H_{\Delta_{1}^{-}}$(voir le lemme 2.4) est un feuilletage produit et que toute géodésique de lumière qui ne coupe pas $\Delta_{1}^{-}$est une transversale totale pour $H_{\Delta_{1}^{-}}$. La projection le long des feuilles définit une submersion $\pi_{\Delta_{1}^{-}}$de $\Omega_{\Delta_{1}^{-}}$ $\operatorname{sur} \Delta_{1}^{-}$.

D'après le lemme 2.4, il existe un difféomorphisme qui envoie l'ouvert $\Omega_{\Delta_{1}^{-}}$sur le produit $\mathbf{S}^{1} \times \mathbf{R}^{n-1}$. De plus, ce difféomorphisme identifie $\Delta_{1}^{+}$à $\mathbf{S}^{1} \times\{0\}$ et les feuilles de $H_{\Delta_{1}^{-}}$aux $\{\theta\} \times \mathbf{R}^{n-1}$. On s'intéresse à présent au domaine fondamental $D$. Chaque surface $\Sigma_{i}^{ \pm}$est transverse à toutes les feuilles de $H_{\Delta_{1}^{-}}$. En effet ces dernières sont de type lumière et ne peuvent pas avoir de points de tangeance avec une hypersurface de signature lorentzienne. Ainsi, il existe un difféomorphisme qui envoie $D$ sur le produit $\mathbf{S}^{1} \times X_{g}$ où $X_{g}$ désigne la sphère $\mathbf{S}^{n-1}$ à laquelle on a ôté $2 g$ disques ouverts disjoints $D_{1}^{ \pm}, \ldots, D_{g}^{ \pm}$. On appelle $S_{1}^{ \pm}, \ldots, S_{g}^{ \pm}$les frontières de ces disques. Les hypersurfaces $\Sigma_{i}^{+}$(resp. $\Sigma_{i}^{-}$) sont identifiées par ce difféomorphisme aux $S^{1} \times S_{i}^{+}$(resp. $\left.S^{1} \times S_{i}^{-}\right)$. La variété $M$ est obtenue en recollant les $S^{1} \times S_{i}^{-}$avec les $S^{1} \times S_{i}^{+}$via les applications $s_{i}:(\theta, x) \mapsto\left(t_{i}(\theta, x), r_{i}(\theta, x)\right)$. Comme les $s_{i}$ sont supposées être dans la composante neutre de $P O(2, n)$, les applications $t_{i}(\theta, x)$ sont homotopes à la projection sur $S^{1}$. Ainsi, les $s_{i}$ sont homotopes aux applications de recollement $(\theta, x) \mapsto\left(\theta, r_{i}(\theta, x)\right.$ ) (où les $r_{i}(\theta, x)$ dépendent différentiablement de $\left.\theta\right)$. Ainsi, $M$ a toujours une structure produit de $S^{1}$ par la variété obtenue à partir de $M_{g}$ en recollant deux à deux les $S_{i}^{+}$et $S_{i}^{-}$. On reconnaît là la somme connexe de $g-1$ copies de $\mathbf{S}^{1} \times \mathbf{S}^{n-2}$.

La topologie de la variété $M=\Omega_{\Gamma} / \Gamma$ dépend uniquement du nombre de générateurs et pas de la configuration des $\Delta_{i}^{ \pm}$.

4.4. Une condition suffisante d'essentialité. Contrairement au cadre riemannien, on ne peut pas prouver qu'une structure lorentzienne conforme sur une variété compacte est essentielle en montrant la non compacité de son groupe conforme. En effet, il existe des exemples de variétés lorentziennes compactes dont le groupe d'isométries lorentziennes est non compact et coïncide avec le groupe conforme. Aussi, pour montrer qu'une structure lorentzienne conforme est essentielle, nous prouverons souvent qu'elle est fortement essentielle, c'est-à-dire que son groupe conforme ne peut préserver aucune mesure absolument continue par rapport à la mesure de Lebesgue.

On se place dans la base $\left(e_{1}, \ldots, e_{n+2}\right)$ et on considère les deux flots de $O(2, n)$ suivants : 


$$
\phi^{t}=\left(\begin{array}{ccccc}
1 & t & & & \\
0 & 1 & & & \\
& & I_{n-2} & & \\
& & & 1 & t \\
& & & 0 & 1
\end{array}\right) \quad \psi^{t}=\left(\begin{array}{lllll}
e^{t} & & & & \\
& e^{-t} & & & \\
& & I_{n-2} & & \\
& & & e^{t} & \\
& & & & e^{-t}
\end{array}\right)
$$

Pour des raisons qui apparaîtront plus claires en section 4.6, on appelle le flot $\phi^{t}\left(\right.$ resp. $\left.\psi^{t}\right)$ ainsi que tout flot qui lui est conjugué dans $P O(2, n)$ un flot de type horocyclique (resp. de type géodésique).

En effectuant une décomposition de Cartan de la matrice $\left(\begin{array}{ll}1 & t \\ 0 & 1\end{array}\right)$ dans $S L_{2}(\mathbf{R})$, on voit aisément que $\phi^{t}$ admet une dynamique à distorsions équilibrées, dont le cercle attracteur et répulseur coïncident avec le cercle $\Delta_{0}$ des points fixes de $\phi^{t}$.

Quant à $\psi^{t}$, il admet clairement une dynamique à distorsions équilibrées. Ses cercles attracteurs et répulseurs $\Delta^{+}$et $\Delta^{-}$sont disjoints et leur réunion constitue l'ensemble des points fixes de $\psi^{t}$.

Les flots horocycliques et géodésiques fournissent un bon moyen pour montrer qu'une structure sur $M$ est fortement essentielle, donc essentielle, comme le montre la :

Soit $M$ une variété munie d'une structure lorentzienne conformément plate. Si le groupe d'holonomie $H$ contient un flot $\phi^{t}$ de type horocyclique (resp. géodésique) dans son centralisateur et que $\Omega=\delta(\tilde{M})$ contient des points fixes de $\phi^{t}$, alors il existe sur $M$ un flot conforme $\bar{\phi}^{t}$ fortement essentiel, qui s'envoie sur $\phi^{t}$ par le morphisme d'holonomie.

Proof. On traîte le cas où $\phi^{t}$ est de type horocyclique (la démonstration s'adapte sans problème pour un flot de type géodésique).

Grâce à l'application développante $\delta, \phi^{t}$ induit sur $M$ un flot conforme $\bar{\phi}^{t}$. On appelle $\Delta_{0}$ le cercle attracteur et répulseur de $\phi^{t}$ et on choisit un petit ouvert $U$ de $\tilde{M}$ tel que les applications $\delta$ et $\pi$ ( $\pi$ désigne l'application de revêtement de $\tilde{M}$ sur $M$ ) soient toutes deux des difféomorphismes de $U$ sur leurs images respectives. On demande de plus à ce que $\delta(U)$ contienne des points de $\Delta_{0}$. Il existe alors un difféomorphisme conforme $\bar{\delta}$ de $\pi(U)$ dans $\delta(U)$ tel que $\bar{\delta} \circ \bar{\phi}^{t}=\phi^{t} \circ \bar{\delta}$ partout où cette expression a un sens. Rappelons que la dynamique de $\phi^{t}$ est à distorsions équilibrées et d'après la proposition 3.2 , il existe une submersion $\pi_{+}$de $\Omega_{\Delta_{0}}$ sur $\Delta_{0}$ telle que pour tout $x \in \Omega_{\Delta_{0}}$, on ait $\lim _{t \rightarrow+\infty} \phi^{t}(x)=\pi_{+}(x)$. On considère alors un petit intervalle $I$ de $\Delta_{0}$ contenu dans $\delta(U)$. On choisit $V$ un ouvert précompact inclus dans $U \cap \pi_{+}^{-1}(I)$. On doit alors avoir $\lim _{t \rightarrow+\infty} \phi^{t}(V) \subset I$. Si $\bar{I}$ et $\bar{W}$ désignent les images de $I$ et $W$ par $\bar{\delta}^{-1}$, on obtient également que $\lim _{t \rightarrow+\infty} \bar{\phi}^{t}(\bar{V}) \subset \bar{I}$. On conclut que $\bar{\phi}^{t}$ ne peut préserver une mesure $\mu$ absolument continue 
par rapport à la mesure de Lebesgue sur $M$. Si c'était le cas, on devrait avoir $\mu\left(\bar{\phi}^{t}(\bar{V})\right)=\mu(\bar{V})$ pour tout $t$, mais comme $\mu(\bar{I})=0$ et $\mu(\bar{V}) \neq 0$, on aboutirait à une absurdité.

4.5. Démonstration du théorème 1. Si $\Gamma$ désigne un groupe de Schottky lorentzien comme dans les sections précédentes, nous allons montrer que la structure $\Omega_{\Gamma} / \Gamma$ est essentielle.

Pour cela, considérons les deux flots $\phi^{t}=\rho_{D}\left(\hat{\phi}^{t}\right)$ et $\psi^{t}=\rho_{D}\left(\hat{\psi}^{t}\right)$ où $\hat{\phi}^{t}=\left(\begin{array}{cc}1 & t \\ 0 & 1\end{array}\right)$ et $\hat{\psi}^{t}=\left(\begin{array}{cc}e^{t} & 0 \\ 0 & e^{-t}\end{array}\right)$. Leurs expressions matricielles montrent immédiatement que $\phi^{t}$ et $\psi^{t}$ sont de type horocyclique et géodésique respectivement. De plus, les points fixes de $\phi^{t}$ constituent une géodésique de lumière $\Delta_{0}$ située dans le feuilletage $F_{2}$ de $\mathbf{T}_{0}$. Le flot $\psi^{t}$ a quant à lui deux géodésiques de lumière de points fixes, $\Delta_{0}^{+}$et $\Delta_{0}^{-}$, elles aussi dans le feuilletage $F_{2}$. En particulier, $\Delta_{0}, \Delta_{0}^{+}$et $\Delta_{0}^{-}$sont toutes trois transverses à $\Lambda_{\Gamma}$ et par conséquent, elles rencontrent l'ouvert $\Omega_{\Gamma}$.

D'autre part, comme $\rho_{D}\left(S L_{2}(\mathbf{R})\right)$ centralise $\Gamma$, les flots $\phi^{t}$ et $\psi^{t}$ induisent deux flots $\bar{\phi}^{t}$ et $\bar{\psi}^{t}$ sur $\Omega_{\Gamma} / \Gamma$. Il n'y a plus quà appliquer la proposition 4.4 pour conclure. Lorsque l'on fixe le nombre $g$ de générateurs, mais que l'on choisit des groupes $\hat{\Gamma}$ non conjugués entre eux, on obtient une infinité de structures conformes essentielles non équivalentes sur $\mathbf{S}^{1} \times$ $\left(\mathbf{S}^{1} \times \mathbf{S}^{n-2}\right)^{(g-1) \sharp}$.

Le groupe conforme de toutes ces structures est, à indice fini près, le produit $S L_{2}(\mathbf{R}) \times O(n-2)$.

Des constructions analogues sont possibles en toute signature pseudoriemannienne, toujours en utilisant des groupes de Schottky. Le théorème de Ferrand-Obata est donc très particulier au contexte riemannien.

\subsection{Interprétation géométrique des exemples précédents en}

dimension 3. En dimension 3, l'ouvert $\mathbf{E i n}_{3} \backslash \mathbf{T}_{0}$ est une composante Antide-Sitter que l'on note (abusivement) $\mathbf{A d S}_{3}$. Le groupe $\Gamma$ agit proprement (mais pas cocompactement) sur $\mathbf{A} \mathbf{d} \mathbf{S}_{3}$ et le quotient $U$ (qui est un ouvert de $\left.M=\Omega_{\Gamma} / \Gamma\right)$ s'identifie au fibré unitaire tangent à la surface hyperbolique ouverte $\Sigma=\mathbf{H}^{2} / \hat{\Gamma}$. En restriction à $U$, les flots $\bar{\phi}^{t}$ et $\bar{\psi}^{t}$ sont respectivement le flot horocyclique et le flot géodésique sur $T^{1} \Sigma$. D'autre part, l'action de $\Gamma$ sur $\mathbf{T}_{0} \cap \Omega_{\Gamma}$ est propre et cocompacte. Le quotient consiste en un nombre fini de tores $T_{1}, \ldots, T_{s}$ et on a $U=M \backslash T_{1} \cup \ldots \cup T_{s}$.

On peut voir $M$ comme la compactification conforme du fibré unitaire tangent à la surface $\Sigma$. Les flots horocycliques et géodésiques, qui préservent une métrique lorentzienne sur $T^{1} \Sigma$, s'étendent en deux flots conformes sur $M$ qui sont fortement essentiels.

4.7. Exemples de structures essentielles non kleiniennes. On se propose de montrer à présent le théorème 1 .

Nous nous plaçons en dimension 3 et considérons $\Gamma=\left\langle\gamma_{1}, \ldots, \gamma_{g}\right\rangle$, un groupe 
de Schottky lorentzien à $g$ générateurs agissant sur $\mathbf{E i n}_{3}$. On fait encore l'hypothèse que $\Gamma$ est contenu dans la composante neutre de $P O(2, n)$. En appliquant le théorème 4.3, on obtient que le quotient $M=\Omega_{\Gamma} / \Gamma$ est difféomorphe au produit $\mathbf{S}^{1} \times \Sigma_{g}$ où $\Sigma_{g}$ désigne le tore à $g$ trous. La structure conformément plate obtenue sur $M=\mathbf{S}^{1} \times \Sigma_{g}$ peut se voir à la fois comme une $\left(\operatorname{Conf}\left(\operatorname{Ein}_{3}\right), \mathbf{E i n}_{3}\right)$-structure et comme une $\left(\operatorname{Conf}\left(\widetilde{\operatorname{Ein}_{3}}\right), \widetilde{\operatorname{Ein}_{3}}\right)$ structure. On désigne par $\pi$ la projection de $\widetilde{\operatorname{Ein}}_{3}$ sur $\mathbf{E i n}_{3}$ et $\bar{\pi}$ la projection de $\operatorname{Conf}\left(\widetilde{\operatorname{Ein}}_{3}\right)$ sur $\operatorname{Conf}\left(\operatorname{Ein}_{3}\right)$ associées. Les applications développantes $\tilde{\delta}$ et $\delta$ sont liées par la relation $\delta=\pi \circ \tilde{\delta}$ et les morphismes d'holonomie vérifient $\rho=\bar{\pi} \circ \tilde{\rho}$. On commence par expliciter le morphisme $\rho$, qui est plus facile à décrire. Les groupes fondamentaux $\pi_{1}(M)$ et $\pi_{1}\left(\Omega_{\Gamma}\right)$ agissent sur $\tilde{M}$ comme deux groupes $\tilde{\Gamma}$ et $\Gamma^{\prime}$ de sorte que $M=\tilde{M} / \tilde{\Gamma}$ et $\Omega_{\Gamma}=\tilde{M} / \Gamma^{\prime}$. On a alors la suite exacte:

$$
1 \rightarrow \Gamma^{\prime} \rightarrow \tilde{\Gamma} \rightarrow \Gamma \rightarrow 1
$$

Le morphisme d'holonomie $\rho$ n'est autre que la projection de $\tilde{\Gamma}$ sur $\Gamma$. On se fixe une fois pour toutes un point base $x_{0}$ de $M$ et $q_{0}$ un point de $\Omega_{\Gamma}$ dans la fibre de $x_{0}$. Les éléments de $\pi_{1}(M)$ qui sont dans le noyau du morphisme d'holonomie sont exactement ceux qui se remontent dans $\Omega_{\Gamma}$ en des lacets basés en $p_{0}$. On peut alors montrer:

Il existe des générateurs $z, a_{1}, b_{1}, \ldots, a_{g}, b_{g}$ de $\pi_{1}(M)$ ( $z$ est un élément central et $\left.\prod_{i=1}^{g}\left[a_{i}, b_{i}\right]=1\right)$ tels que:

$\rho(z)=i d$

$\rho\left(a_{i}\right)=i d(i \in\{1, \ldots, g\})$

$\rho\left(b_{i}\right)=\gamma_{i}(i \in\{1, \ldots, g\})$

Proof. On appelle $D$ un domaine fondamental du groupe $\Gamma$, et on peut supposer que $p_{0}$ appartient à $D$. Comme nous l'avons vu lors de la proposition $4.3, D$ est homéomorphe au produit $\mathbf{S}^{1} \times X_{g}$ où $X_{g}$ désigne la sphère $\mathbf{S}^{2}$ privée de $2 g$ disques ouverts $D_{i}^{ \pm}$. Le groupe fondamental $\pi_{1}(D)$ est donc isomorphe au produit de $\mathbf{Z}$ par un groupe libre à $2 g$ générateurs. On choisit $s, t_{1}, \ldots, t_{g}, t_{g+1}, \ldots, t_{2 g}$ le système de générateurs suivant:

- $s$ est central. C'est un lacet qui part de $p_{0}$ et fait une fois le tour du facteur $\mathbf{S}^{1}$ dans le sens positif.

- pour $i \in\{1, \ldots, g\}, t_{i}$ est le lacet qui part de $p_{0}$ et fait une fois le tour de $D_{i}^{-}$dans le sens positif.

- pour $i \in\{g+1, \ldots, 2 g\}, t_{i}$ est le lacet qui part de $p_{0}$ et fait une fois le tour de $D_{i}^{+}$dans le sens positif.

On projette les lacets $s, t_{1}, \ldots, t_{g}$ sur $M$ et on appelle leurs images $z, a_{1}, \ldots, a_{g}$. D'autre part, on considère $g$ lacets $b_{i}(i \in\{1, \ldots, g\})$ basés en $x_{0}$ dont les remontés $\tilde{b}_{i}$ dans $\Omega_{\Gamma}$ à partir de $p_{0}$ vérifient $\tilde{b}_{i}(1)=\gamma_{i} . p_{0}$. Alors $z, a_{1}, \ldots, a_{g}, b_{1}, \ldots, b_{g}$ est un système de générateurs pour $\pi_{1}(M)$. De plus, comme $z, a_{1}, \ldots, a_{g}$ se relèvent dans $\Omega_{\Gamma}$ en des lacets basés en $p_{0}$, ils sont tous dans le noyau de $\rho$. D'autre part, $\rho\left(b_{i}\right)=\gamma_{i}$ pour tout $i$ dans $\{1, \ldots, g\}$. 
Comme le groupe $\Gamma$ est dans la composante neutre de $P O(2, n)$, chaque $\gamma_{i}$ est le temps 1 d'un groupe à un paramètre $\gamma_{i}^{t}$. Les groupes $\gamma_{i}^{t}$ se remontent en groupes $\tilde{\gamma}_{i}^{t}$ de $\operatorname{Conf}\left(\widetilde{\operatorname{Ein}}_{3}\right)$. D'autre part, le centre de $\widetilde{\operatorname{Ein}}_{3}$ est infini cyclique, engendré par un élément $\zeta$ et on a $\boldsymbol{\operatorname { E i n }}_{3}=\widetilde{\operatorname{Ein}}_{3} /\langle\zeta\rangle$. On peut maintenant décrire la représentation $\tilde{\rho}$ :

$-\tilde{\rho}(z)=\zeta^{k}$ pour un certain entier $k$.

$-\tilde{\rho}\left(a_{i}\right)=\zeta^{m_{i}}$ (pour certains entiers $m_{i}$ ).

$-\tilde{\rho}\left(b_{i}\right)=\zeta^{n_{i}} \tilde{\gamma}_{i}^{1}$ (avec $n_{i}$ entiers).

Nous utilisons maintenant un théorème de déformation, que l'on attribue à Ehresmann (voir $[\mathrm{Th}])$ :

[Principe de déformation] Soit $M$ une variété compacte munie d'une $(G, X)$ structure de morphisme d'holonomie $\rho_{0}$. Il existe alors un voisinage $U$ de $\rho_{0}$ dans $\operatorname{Hom}\left(\pi_{1}(M), G\right)$ tel que tout $\rho$ de $U$ est réalisé comme l'holonomie d'une $(G, X)$-structure sur $M$.

On considère la famille $\tilde{\rho}_{u}$ de représentations de $\pi_{1}(M)$ dans $\operatorname{Conf}\left(\widetilde{\operatorname{Ein}_{3}}\right)$ définie par :

$-\tilde{\rho}_{u}(z)=\zeta^{k}$.

$-\tilde{\rho}_{u}\left(a_{i}\right)=\zeta^{m_{i}} \tilde{\gamma}_{i}^{u}$

$-\tilde{\rho}_{u}\left(b_{i}\right)=\zeta^{n_{i}} \tilde{\gamma}_{i}^{1}$.

Pour $u$ petit, le théorème 4.7 assure que $\tilde{\rho}_{u}$ est l'holonomie d'une structure conformément plate sur $\mathbf{S}^{1} \times \Sigma_{g}$. Nous affirmons que pour $u$ petit, cette structure est encore essentielle. En effet, soit $H_{u}=\bar{\pi} \circ \tilde{\rho}_{u}\left(\pi_{1}(M)\right)$ le groupe d'holonomie dans $\operatorname{Conf}\left(\mathbf{E i n}_{3}\right)$ de la structure déformée. Le flot $\phi^{t}$ de la section 4.5 centralise $H_{u}$. D'autre part, pour $u$ petit le domaine fondamental $D_{u}$ pour l'action de $H_{u}$ est très proche du domaine fondamental $D$ de l'action de $H_{0}=\Gamma$. Ainsi, comme $D$ contient des points fixes de $\phi^{t}$ dans son intérieur, $D_{u}$ fait de même. La proposition 4.4 assure alors que la structure déformée reste essentielle. Pour finir, si on choisit $u$ irrationnel, $\tilde{\rho}_{u}\left(\pi_{1}(M)\right)$ n'est pas discret, et par conséquent, la structure déformée n'est plus kleinienne.

Le procédé que nous venons de décrire semble très particulier à la dimension 3, et on peut se demander si la situation se rigidifie en dimension supérieure. Autrement dit, les structures essentielles sont-elles toutes kleiniennes en dimension $\geq 4$ ?

Remerciements. Nous voudrions remercier le référé pour ses remarques.

\section{REFERENCES}

[A] D.Alekseevski - Selfsimilar lorentzian manifolds. Ann. Global Anal. Geom. Vol. 3, No. 1 (1985), 59-84.

[D'AG] G.D'AmBrA; M.Gromov Lectures on transformation groups: geometry and dynamics. Surveys in differential geometry (Cambridge, MA, 1990), 19-111, Lehigh Univ., Bethlehem, PA, 1991. 
[B] Y.Benoist - Proprits asymptotiques des groupes linaires. Geom. Funct. Anal. 7 (1997), no. 1, 1-47.

[CK] M.CAhen; Y.Kerbrat - Domaines symtriques des quadriques projectives. J. Math. Pures Appl. (9) 62 (1983), no. 3, 327-348.

[dlH] P.DE LA HARPE - Free groups in linear groups. Enseign. Math. (2) 29 (1983), no. 1-2, 129-144.

[Fe2] J.FERRAND - Transformations conformes et quasiconformes des varits riemanniennes; application la dmonstration d'une conjecture de A. Lichnerowicz. C. R. Acad. Sci. Paris Sr. A-B 269 (1969)

[Fr1] C.FrAnCES - Géométrie et dynamique lorentziennes conformes. Thèse. 2002. http://www.umpa.ens-lyon.fr/ cfrances/

[Fr2] C.FRANCES - Lorentzian Kleinian groups. preprint. http://www.umpa.enslyon.fr/ cfrances/

[FT] C.Frances; C.TARquini - Autour du thorme de Ferrand-Obata. Ann. Global Anal. Geom. 21 (2002), no. 1, 51-62

[HE] S.W.HAWking; G.F.R.Ellis - The large scale structure of space-time. Cambridge Monographs on Mathematical Physics, No. 1. Cambridge University Press, London-New York, 1973.

[IW] A.IozzI; D.WITTE - Cartan-decomposition subgroups of $\mathrm{SU}(2, n)$. J. Lie Theory 11 (2001), no. 2, 505-543.

[KR] W.Khnel; H.B.RAdemacher - Essential conformal fields in pseudoRiemannian geometry. J. Math. Pures Appl. (9) 74 (1995), no. 5, 453-481.

[Ma] B.MASKIT - Kleinian groups. Grundlehren der Mathematischen Wissenschaften , 287. Springer-Verlag, Berlin, 1988.

[Ob] M.OBATA - The conjectures on conformal transformations of Riemannian manifolds. J. Differential Geometry 6 (1971/72), 247-258.

[P] R.PEnRose - Conformal treatment of infinity. in Relativit, Groupes et Topologie (Lectures, Les Houches, 1963 Summer School of Theoret. Phys., Univ. Grenoble) pp. 563-584 Gordon and Breach, New Ann. Global Anal. Geom. 21 (2002), no. 1, 51-62York.

[SV] J.Seade; A.VerJovsky - Higher dimensional complex Kleinian groups. Math. Ann. 322 (2002), no. 2, 279-300.

[Th] W.Thurston - Three dimensional geometry and topology. Vol 1.- Princeton University Press, 1997. Edited by Silvio Levy 